\title{
Gating deficits in isolation-reared rats are correlated with alterations in protein expression in nucleus accumbens
} \author{
Alessio Soggiu, $\mathscr{~ I ~ S a l v a t o r e ~ P i s a n u , ~} \mathscr{~ I ~ A n g e l a ~ A m o r e s a n o , * * ~ A n d r e a ~ C a r p e n t i e r i * * ~ a n d ~}$ \\ Paola Devotoł’ \\ *Istituto Sperimentale Italiano "L. Spallanzani”, Milano, Italy \\ $\dagger$ Department of Cardiovascular and Neurological Science, University of Cagliari, Cagliari, Italy \\ $\$$ Tourette Syndrome Centre, University of Cagliari, Cagliari, Italy \\ $\S$ “B.B. Brodie” Department of Neuroscience, University of Cagliari, Cagliari, Italy \\ IIProteotech srl, Sardegna Ricerche Science Park, Pula, Italy \\ **Department of Organic Chemistry and Biochemistry, University of Naples, Naples, Italy
}

Paola Roncada, ${ }^{*}$ Marco Bortolato, $\uparrow \ddagger$ Roberto Frau, $\$ \S$ Pierluigi Saba, $\S$ Giovanna Flore, $\uparrow \ddagger$

\begin{abstract}
The isolation-rearing (IR) paradigm, consisting of the social deprivation for 6-9 weeks after weaning, induces a spectrum of aberrant behaviors in adult rats. Some of these alterations such as sensorimotor gating deficits are reminiscent of the dysfunctions observed in schizophrenia patients. Although gating impairments in IR rats have been linked to impairments in the cortico-mesolimbic system, the specific molecular mechanisms underlying this relation are unclear. To elucidate the neurochemical modifications underlying the gating disturbances exhibited by IR rats, we compared their pre-pulse inhibition (PPI) of the acoustic startle reflex with that of socially reared (SR) controls, and correlated this index to the results of proteomic analyses in prefrontal cortex and nucleus accumbens from both groups. As expected, IR rats exhibited significantly lower startle amplitude and PPI than their SR counterparts. Following behavioral testing, IR and SR rats were killed and protein expression profiles of their brain regions were examined using two-dimensional electrophoresis
\end{abstract}

based proteomics. Image analysis in the Coomassie bluestained gel revealed that three protein spots were differentially expressed in the nucleus accumbens of IR and SR rats. Mass spectrometry (matrix-assisted laser desorption ionization-time of flight and MS/MS) identified these spots as heat shock protein 60 (HSP60), $\alpha$-synuclein ( $\alpha$-syn), and 14-3-3 protein $\zeta /$ $\delta$. While accumbal levels of HSP60 was decreased in IR rats, $\alpha$-syn and 14-3-3 proteins were significantly increased in IR in comparison with SR controls. Notably, these two last alterations were significantly correlated with different loudness intensity-specific PPI deficits in IR rats. In view of the role of these proteins in synaptic trafficking and dopaminergic regulation, these findings might provide a neurochemical foundation for the gating alterations and psychotic-like behaviors in IR rats.

Keywords: $14-3-3$ protein, $\alpha$-synuclein, heat shock protein 60 , isolation rearing, pre-pulse inhibition, proteomics.

J. Neurochem. (2009) 108, 611-620.
Most current animal models of schizophrenia are based on invasive paradigms or pharmacologically induced states. These non-naturalistic approaches, albeit useful to represent specific aspects of the disorder, are poorly suitable for the study of neurochemical and functional imbalances in psychosis. An experimental model that more closely captures the neurobiological relationship between environmental stress and schizophrenia is the isolation-rearing (IR)
Received June 9, 2008; revised September 11, 2008; accepted November 6, 2008.

Address correspondence and reprint requests to Dr. Paola Devoto, $\mathrm{PhD}$, "B.B. Brodie" Department of Neuroscience, Cittadella Universitaria, SS 554, Monserrato I-09042, Italy. E-mail: pdevoto@unica.it

Abbreviations used: $\alpha$-syn, $\alpha$-synuclein; ACN, acetonitrile; DA, dopamine; HSP, heat shock protein; IR, isolation rearing; MALDI, matrix-assisted laser desorption ionization; $\mathrm{mPFC}$, medial prefrontal cortex; MS, mass spectrometry; NAcc, nucleus accumbens; PPI, pre-pulse inhibition; SR, socially reared. 
paradigm (for a review, see Fone and Porkess 2008). IR consists of subjecting rats to the deprivation of social interactions for 6-9 weeks after weaning, a critical phase of rodent life span characterized by the development of social play (Einon and Morgan 1977). As a result of this manipulation, IR rats exhibit a spectrum of persistent behavioral alterations in adulthood, including hyperactivity, increased responsiveness to psychostimulants, perseverative behaviors, cognitive deficits, and disruption of sensorimotor gating (Weiss and Feldon 2001). These abnormalities are paralleled by multiple neurochemical changes, such as aberrant dopamine (DA), serotonin, and noradrenaline levels in prefrontal cortex and nucleus accumbens (NAcc) (Fulford and Marsden 1998; Brenes et al. 2008) and altered mesocortical transmission (Peters and O'Donnell 2005).

Some of these behavioral and neurochemical disturbances are typically reminiscent of those observed in schizophrenia patients. In particular, several lines of evidence suggest that the deficit in pre-pulse inhibition (PPI) of the acoustic startle may be the behavioral disturbance in IR rats with the highest validity as a model of psychosis (Geyer et al. 1993). PPI is the reduction in the startling reaction elicited by a strong sensory stimulus that occurs when the latter is immediately preceded by a weaker signal. PPI deficits are reflective of gating abnormalities and are typically observed in schizophrenia patients (Braff et al. 1992). In contrast with other antipsychotic-resistant alterations in IR rats (Garzón and Del Río 1981), the PPI disruption produced by this manipulation is efficiently and selectively reversed by antipsychotic agents (Bakshi et al. 1998; Binder et al. 2001). Even though several investigations have shed light on some of the neural substrates of PPI impairments in IR rats, the biological alterations generating this complex phenomenon still remain to be investigated.

In recent years, technical developments in the field of proteomics have generated advances in our understanding of protein expression, function, and organization in signaling processes and regulatory networks, providing deeper insight in how cellular proteomes are regulated in the nervous system. Two-dimensional electrophoresis offers a powerful and efficient approach to measure abundant protein changes in normal and pathological states. The major advantage of two-dimensional electrophoresis lies in its potential to simultaneously resolve thousands of proteins, reveal their molecular weight and $\mathrm{pI}$, and reflect changes in protein expression and isoforms (Gorg et al. 2004; Carrette et al. 2006a). In the past decade, many studies based on 2Dpolyacrylamide gel electrophoresis followed by mass spectrometry (MS) have been published on different animal models (mouse and rat) (Fountoulakis et al. 1999, 2005; Fountoulakis 2004). Fountoulakis et al. (1999) described a two-dimensional database of rat brain proteins including approximately 200 different proteins. The establishment of comprehensive brain proteome maps proves to be a useful reference database for the study of changes in protein expression levels associated with psychiatric and neurodegenerative disorders (Johnston-Wilson et al. 2000; Teunissen and Scheltens 2007) as well as development and aging (Carrette et al. 2006b). To identify the nature of the changes which underlie psychotic-like abnormalities in IR rats in this study we analyzed the correlation between their PPI alterations and the changes in protein expression of their medial prefrontal cortex (mPFC) and NAcc, as assessed by a proteomic analysis.

\section{Materials and methods}

\section{Isolation rearing}

Experiments were approved by the local ethical committee and performed according to the Guidelines for Care and Use of Experimental Animals of the European Union (EEC Council 86/ 609; D.L. 27/01/1992, n. 116). Sprague-Dawley dams were received from the supplier (Harlan Italy, S. Pietro al Natisone, Italy) each one with 10 pups aged 8 days on arrival. At postnatal day 21, male rats were weaned and assigned to either IR or socialrearing (SR) groups, ascertaining that littermates were equally distributed across the two groups (to avoid litter effects). When more than two littermates were designated to SR groups, they were not placed together in the same cage. IR rats were reared individually in plastic cages, while SR rats were housed four per cage. Animals were disturbed only for cleaning purposes, which consisted of changing the cage once a week for IR and twice a week for SR animals. Both groups were housed in the same room so that IR rats maintained visual, auditory, and olfactory contact with the other animals. The room was kept under standard conditions of temperature and humidity, and food and water was available ad libitum. Artificial light was on from 8 PM to 8 AM. Experiments were conducted during the light-off phase of the day. After 8 weeks of isolation-rearing, IR and SR animals ( $n=16$ /group, from different litters) were tested for startle and PPI evaluation.

\section{Startle and pre-pulse inhibition}

The apparatus used for detection of startle reflexes (Med Associates, St Albans, VT, USA) consisted of four standard cages placed in sound-attenuated chambers with fan ventilation. Each cage consisted of a Plexiglas cylinder of $9 \mathrm{~cm}$ diameter, mounted on a piezoelectric accelerometric platform connected to an analog-digital converter. Background noise and acoustic bursts were conveyed by two separate speakers, each one properly placed so as to produce a variation of sound within $1 \mathrm{~dB}$ across the startle cage. Both speakers and startle cages were connected to a main PC, which detected and analyzed all chamber variables with specific software. Before each testing session, acoustic stimuli and mechanical responses were calibrated via specific devices supplied by Med Associates.

At 3 days before the experiment, all rats went through a brief baseline startle session. Rats were exposed to a background noise of $70 \mathrm{~dB}$ and, after an acclimatization period of $5 \mathrm{~min}$, were presented with a randomized sequence of twelve 40 -ms bursts of $115 \mathrm{~dB}$, interposed with three trials in which a $82 \mathrm{~dB}$ pre-stimulus preceded the same pulse by $100 \mathrm{~ms}$. On the testing day, each rat was placed in 
a cage for a 5-min acclimatization period with a $70 \mathrm{~dB}$ white noise background, which continued for the remainder of the session. Each session consisted of three consecutive sequences of trials (periods). Unlike the first and the third period, during which rats were presented with only five pulse-alone trials of $115 \mathrm{~dB}$, the second period consisted of a pseudorandom sequence of 50 trials, including 12 pulse-alone trials, 30 trials of pulse preceded by 73,76 , or $82 \mathrm{~dB}$ pre-pulses (10 for each level of pre-pulse loudness), and eight no stimulus trials, where only the background noise was delivered. Inter-trial intervals were selected randomly between 10 and $15 \mathrm{~s}$.

\section{Proteomic analysis}

\section{Sample preparation}

After 7 days from PPI disruption evaluation, rats were killed by decapitation, and whole brains were rapidly removed, washed with phosphate-buffered saline plus protease and phosphatase inhibitors and areas of interest were dissected, according to the method described by Heffner et al. (1980). Briefly, brains were placed in a cutting block kept on ice, a razor blade was inserted tangential to the most posterior aspect of the olfactory tubercle at the level of the anterior commissure, and then three more anterior blades were inserted at $1.5-\mathrm{mm}$ interval. NAcc and $\mathrm{mPFC}$ were dissected from the second and third slice, respectively (Fig. 1). Each sample was immediately added with protease inhibitor cocktail (Sigma, St Louis, MO, USA) and phosphatase inhibitor cocktail 1 and 2 (Sigma) according to the manufacturer's instructions, flash frozen in liquid nitrogen, and stored at $-80^{\circ} \mathrm{C}$ until further use. Prior to their use, the tissue was weighed and suspended $(1: 10, \mathrm{w} / \mathrm{v})$ in a solution of $7 \mathrm{M}$ urea, $2 \mathrm{M}$ thiourea, $2 \%$ 3-[(3-Cholamidopropyl)dimethylammonio-1-propanesulfonate (CHAPS), 2\% Triton X-100, $15 \mathrm{mM}$ Tris, 2\%(v/v) Ampholine $\mathrm{pH} 3.5-10$ and $65 \mathrm{mM}$ dithiothreitol. The suspension was homogenized under mechanic stirring for $3 \mathrm{~h}$ and was then centrifuged at $14000 \mathrm{~g}$ for $30 \mathrm{~min}$ to remove cellular debris and insoluble materials. The resulting supernatant was treated with $100 \mathrm{mM}$ hydroxyethyl disulfide for $1 \mathrm{~h}$

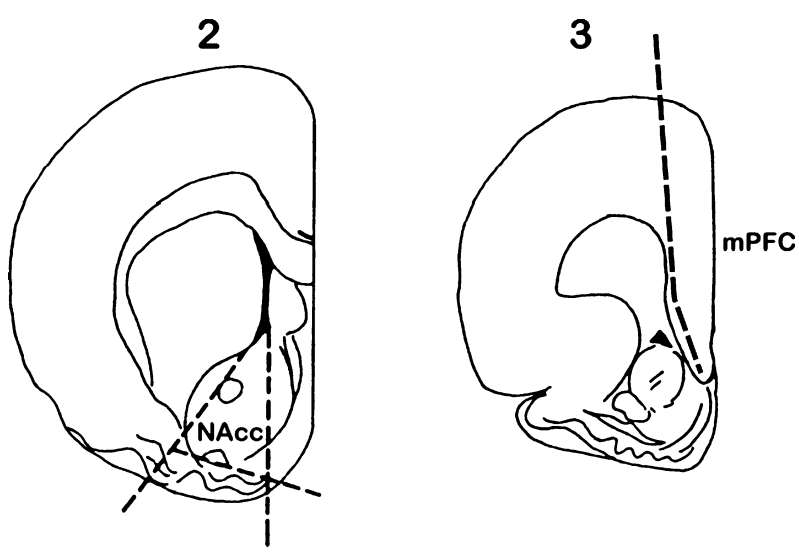

Fig. 1 Schematic diagram showing the brain areas dissected for proteomic analysis. Slices were cut at $1.5 \mathrm{~mm}$ interval, as described in Materials and methods. Nucleus accumbens (NAcc) and medial prefrontal cortex (mPFC) were dissected from the second and third slice, respectively. at $25^{\circ} \mathrm{C}$ to form mixed disulfides with cysteinyl thiols to reduce the streaks caused by re-oxidation of disulphide bridges. Protein concentrations were determined using the 2D-Quant Kit (GE Healthcare, Uppsala, Sweden).

\section{Two dimensional gel electrophoresis}

For each sample, $7 \mathrm{~cm}$ immobilized $\mathrm{pH}$ gradient strips, $\mathrm{pH} 3-10$ non-linear gradient (GE Healthcare) were rehydrated overnight. An appropriate amount of the solubilized protein sample from each brain area of interest was loaded onto each strip by cup loading. Isoelectrofocusing was then performed on a IPGPHOR 3 apparatus (GE Healthcare) at $20^{\circ} \mathrm{C}$ by a series of increasing voltage 'steps' from 50 to $5000 \mathrm{~V}$, until the total voltage of $100000 \mathrm{~V} \times \mathrm{h}$ was reached. The strips were equilibrated sequentially for $15 \mathrm{~min}$ in equilibration buffer $(50 \mathrm{mM}$ Tris- $\mathrm{HCl}, \mathrm{pH} 8.8,6 \mathrm{M}$ urea, $2 \%$ sodium dodecyl sulfate, $30 \%$ glycerol) containing $1 \%$ dithiothreitol and then $2.5 \%$ iodoacetamide. For the second dimension, proteins were separated by $9-16 \%$ gradient - sodium dodecyl sulphatepolyacrylamide gel electrophoresis. Strips were held in place on top of gels by $0.5 \%$ agarose in running buffer and electrophoresis was performed on a Mini Protean III system (Bio-Rad Laboratories, Hercules, CA, USA) for $15 \mathrm{~min}$ at 50 and $100 \mathrm{~V}$ until tracking dye (bromophenol blue) reached the bottom of the gel. Proteins were visualized by colloidal Coomassie or silver staining. Molecular masses were determined by analyzing standard protein markers that cover the range $10-200 \mathrm{kDa}$ and $\mathrm{pI}$ values are used as given by the supplier of the immobilized $\mathrm{pH}$ gradient strips. Suitable staining and destaining protocols were followed before the gel imaging step. The gels were scanned in a PHAROS FX apparatus (Bio-Rad Laboratories). Four gels were generated for each set of samples to minimize experimental variability and allow for a statistically robust analysis. Image analysis was performed using ImageMaster 2D Platinum v6.0.1 software (GE Healthcare). The highest variable spots were identified by matrix-assisted laser desorption ionization-time of flight (MALDI-TOF) and MS/MS.

\section{In situ digestion}

The analysis was performed on the Coomassie blue-stained spots excised from gels. The excised spots were washed first with acetonitrile $(\mathrm{ACN})$ and then with $0.1 \mathrm{M}$ ammonium bicarbonate. Protein samples were reduced by incubation in $10 \mathrm{mM}$ dithiothreitol for $45 \mathrm{~min}$ at $56^{\circ} \mathrm{C}$. The cysteines were alkylated by incubation in $5 \mathrm{mM}$ iodoacetamide for $15 \mathrm{~min}$ at $25^{\circ} \mathrm{C}$ in the dark. The gel particles were then washed with ammonium bicarbonate and ACN. Enzymatic digestion was carried out with trypsin $(12.5 \mathrm{ng} / \mu \mathrm{L})$ in $50 \mathrm{mM}$ ammonium bicarbonate $\mathrm{pH} 8.5$ at $4^{\circ} \mathrm{C}$ for $4 \mathrm{~h}$. The buffer solution was then removed and a new aliquot of the enzyme/buffer solution was added for $18 \mathrm{~h}$ at $37^{\circ} \mathrm{C}$. A minimum reaction volume enough for the complete rehydratation of the gel was used. Peptides were then extracted by washing the gel particles with $20 \mathrm{mM}$ ammonium bicarbonate and $0.1 \%$ trifluoroacetic acid in $50 \% \mathrm{ACN}$ at $25^{\circ} \mathrm{C}$ and then lyophilised.

\section{MALDI-TOF mass spectrometry}

Positive Reflectron MALDI spectra were recorded on a Voyager DE STR instrument (Applied Biosystems, Framingham, MA, USA). The MALDI matrix was prepared by dissolving $10 \mathrm{mg}$ of $\alpha$-cyano in $1 \mathrm{~mL}$ of ACN/water $(90: 10 \mathrm{v} / \mathrm{v})$. Typically, $1 \mu \mathrm{L}$ of matrix was 
applied to the metallic sample plate and $1 \mu \mathrm{L}$ of analyte was then added. Acceleration and reflector voltages were set up as follows: target voltage at $20 \mathrm{kV}$, first grid at $95 \%$ of target voltage, delayed extraction at $600 \mathrm{~ns}$ to obtain the best signal-to-noise ratios and the best possible isotopic resolution with multipoint external calibration using peptide mixture purchased from Applied Biosystems. Each spectrum represented the sum of 1500 laser pulses from randomly chosen spots per sample position. Raw data were analyzed using the computer software provided by the manufacturers and are reported as monoisotopic masses.

\section{NanoLC mass spectrometry}

A mixture of peptide solution was analyzed by LC-MS analysis using a 4000Q-Trap (Applied Biosystems) coupled to an 1100 nano HPLC system (Agilent Technologies, Wilmington, DE, USA). The mixture was loaded on an Agilent reverse-phase precolumn cartridge (Zorbax $300 \mathrm{SB}-\mathrm{C} 18,5 \times 0.3 \mathrm{~mm}, 5 \mu \mathrm{m}$ ) at $10 \mu \mathrm{L} / \mathrm{min}$ (A solvent $0.1 \%$ formic acid, loading time $5 \mathrm{~min}$ ). Peptides were separated on a Agilent reverse-phase column (Zorbax $300 \mathrm{SB}-\mathrm{C} 18,150 \mathrm{~mm} \times 75 \mu \mathrm{m}, 3.5 \mu \mathrm{m}$ ), at a flow rate of $0.3 \mu \mathrm{L} / \mathrm{min}$ with a $0 \%$ to $65 \%$ linear gradient in $60 \mathrm{~min}$ (A solvent $0.1 \%$ formic acid, $2 \%$ ACN in Milli-Q water; B solvent $0.1 \%$ formic acid, $2 \%$ MQ water in $\mathrm{ACN}$ ). Nanospray source was used at $2.5 \mathrm{kV}$ with liquid coupling, with a declustering potential of $20 \mathrm{~V}$, using an uncoated silica tip from NewObjectives (O.D.: $150 \mu \mathrm{m}$; I.D.: $20 \mu \mathrm{m}$; T.D.: $10 \mu \mathrm{m}$ ). Data were acquired in information-dependent acquisition mode, in which a full scan mass spectrum was followed by MS/MS of the five most abundant ions ( $2 \mathrm{~s}$ each). In particular, spectra acquisition of MS/MS analysis was based on a survey Enhanced MS Scan from 400 to $1400 \mathrm{~m} / \mathrm{z}$ at $4000 \mathrm{amu} / \mathrm{s}$. This scan mode was followed by an Enhanced Resolution experiment for the five most intense ions and then MS2 spectra Enhanced Product Ion (EPI) were acquired using the best collision energy calculated on the bases of $\mathrm{m} / \mathrm{z}$ values and charge state (rolling collision energy) from 100 to $1400 \mathrm{~m} / \mathrm{z}$ at $4000 \mathrm{amu} / \mathrm{s}$. Data were acquired and processed using Analyst software (Applied Biosystems).

\section{MASCOT analysis}

Spectral data were analyzed using Analyst software (version 1.4.1) and MS/MS centroid peak lists were generated using the MASCOT.dll script (version 1.6b9, Matrix Science, London, UK). MS/ MS centroid peaks were threshold at $0.1 \%$ of the base peak. MS/ MS spectra having less than 10 peaks were rejected. MS/MS spectra were searched against Swiss Prot database (2006.10.17 version, Swiss Institute of Bioinformatics, Geneva, Switzerland) using the licensed version of Mascot 2.1 version (Matrix Science, London, UK), after converting the acquired MS/MS spectra in mascot generic file format. The Mascot search parameters were taxonomy rat; allowed number of missed cleavages 2; enzyme trypsin; variable post-translational modifications; methionine oxidation; pyro-glu N-term Q; peptide tolerance $200 \mathrm{ppm}$ and MS/MS tolerance $0.5 \mathrm{Da}$; and peptide charge from +2 to +3 and top 20 protein entries. Spectra with a MASCOT score $<25$ were having low quality and were rejected. The score used to evaluate quality of matches for MS/MS data was higher than 30. However, spectral data were manually validated and contained sufficient information to assign peptide sequence.

\section{Data analysis}

For each animal, the mean startle amplitudes for the session as well as the mean latency to peak values of startle were analyzed with ANOVA, with treatment as a between-subject factor. Startle habituation across the two halves of the session (blocks) was evaluated as \% inter-block reduction (IBR), using the following formula: $100-[($ mean startle amplitude for first block/mean startle amplitude for second block)*100].

The \%PPI was calculated using the following formula: $100-[$ (mean startle amplitude for pre-pulse pulse trials/mean startle amplitude for pulse alone trials)*100] and analyzed by twoway ANOVAs with rearing condition (i.e., isolation-reared vs. groupreared) as a between-subjects factor and trial types as repeated measures.

For proteomic analysis, representative subpopulations of IR and SR rats ( $n=4$ /group) were selected. To minimize sampling errors, selection was performed with disproportionate stratification of the animals in each group, based on ranking of the average \%PPI values and verification of the homoscedasticity of the sampling strata via Bartlett's test. We ascertained that mean \%PPI of the representative samples differed from the average \%PPI of each group by no more than $5 \%$. Statistical effects on startle magnitude, \%IBR, latency to startle peak, and \%PPI and $\triangle \mathrm{PPI}$ were confirmed in the IR and SR subgroups.

Proteomic data were evaluated using Student's $t$-test to assess statistical differences between comparison groups, which contained at least four independent samples. Correlation between behavioral and neurochemical data in IR rats was also studied by means of linear regression analysis. Significance limit was set at 0.05 .

\section{Results}

\section{Startle and PPI analysis}

Startle magnitudes were compared by one-way ANOVA, with rearing condition as variable. Isolation-reared rats displayed a significantly reduced startle amplitude $\left[F_{(1,30)}=7.36, p<\right.$ 0.05, ANOva] (Fig. 2a). Conversely, no difference between the two groups was identified in either latency to startle peak $\left[F_{(1,30)}=1.70, \mathrm{NS}\right]$ or $\% \mathrm{IBR}$ values $\left[F_{(1,30)}=0.85, \mathrm{NS}\right]$ (data not shown).

Percent PPI analysis was performed by a two-way ANOvA design, with rearing condition and pre-pulse levels as factors (Fig. 2c). Statistical analysis detected that \%PPI was significantly lower in isolation-reared rats when compared with group-reared rats $\left[F_{(1,30)}=13.23, p<0.01\right]$. Significant effects were also found for pre-pulse levels $\left[F_{(2,60)}=5.61\right.$, $p<0.01]$. Post hoc scrutiny of these effects detected a significant difference between the \%PPI elicited by $73 \mathrm{~dB}$ in comparison with those generated by $76 \mathrm{~dB}(p<0.05)$ and $82 \mathrm{~dB}(p<0.01)$. Finally, there were no significant pre-pulse levels $\times$ rearing condition interactions $\left[F_{(2,60)}=\right.$ 0.72, NS].

Although littermates were equally distributed in IR and SR groups, to ensure the absence of potential litter-related effects on the comparisons of startle and PPI data, we verified the 
(a)

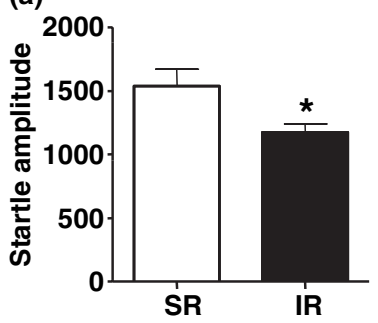

(c)

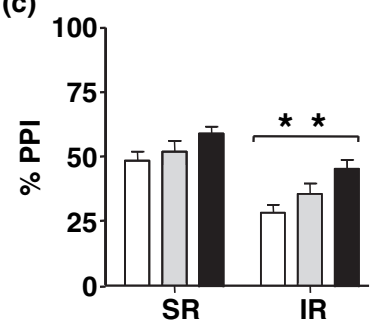

(b)

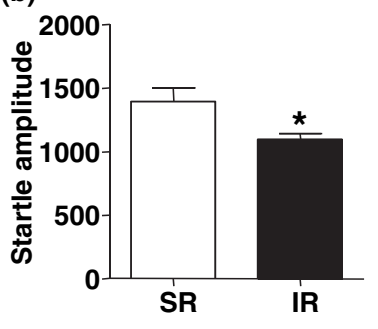

(d)

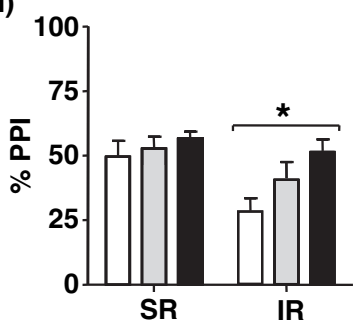

Fig. 2 Startle and pre-pulse inhibition (PPI) analysis in isolationreared rats (IR) and their socially reared controls (SR). Values represent mean \pm SEM. for each group. Pre-pulses are indicated by the intensity corresponding to decibels above background noise. a-c: total groups $(n=16)$; b-d: subgroups selected for proteomic analysis $(n=4) .{ }^{*} p<0.05 ;{ }^{* *} p<0.01$ in comparison to SR. For further details, see 'Startle and PPI analysis' section.

absence of rearing conditions $\times$ litter interactions on all startle and PPI parameters by multiway ANOvA analyses (data not shown).

The analysis of startle and PPI values for the two representative subgroups $(n=4)$, selected by ranking-based disproportionate stratification for proteomic analysis, was performed with the same statistical designs. As shown in Fig. 2b, the selected IR rats displayed a significantly reduced startle amplitude in comparison with SR counterparts $\left[F_{(1,6)}=8.91, p<0.05\right.$, ANOVA $]$ and confirmed no difference in either latency to startle peak $\left[F_{(1,6)}=0.01, \mathrm{NS}\right]$ or \%IBR values $\left[F_{(1,6)}=0.06, \mathrm{NS}\right]$ (data not shown). Furthermore, $\%$ PPI values were significantly lower in IR rats $[\% \mathrm{PPI}$ : $F_{(1,6)}=8.63, p<0.05$ ] (Fig. 2d). Significant effects were found for pre-pulse levels $\left[F_{(2,612)}=4.65, p<0.05\right]$ and for pre-pulse levels $\times$ rearing condition interactions $\left[F_{(2,12)}=\right.$ $4.30, p<0.05]$ in the \%PPI analysis. However, post hoc comparison with Tukey's test did not reveal any significant difference between groups. We further ruled out sampling bias by verifying the absence of significant differences between the four animals selected and the residual animals in each group for each startle or PPI parameter by ANOvA (data not shown).

\section{Proteomic analysis}

About 300 proteins were detected by the 2D ImageMaster software (GE Healthcare, Uppsala, Sweden) on the Coomas-

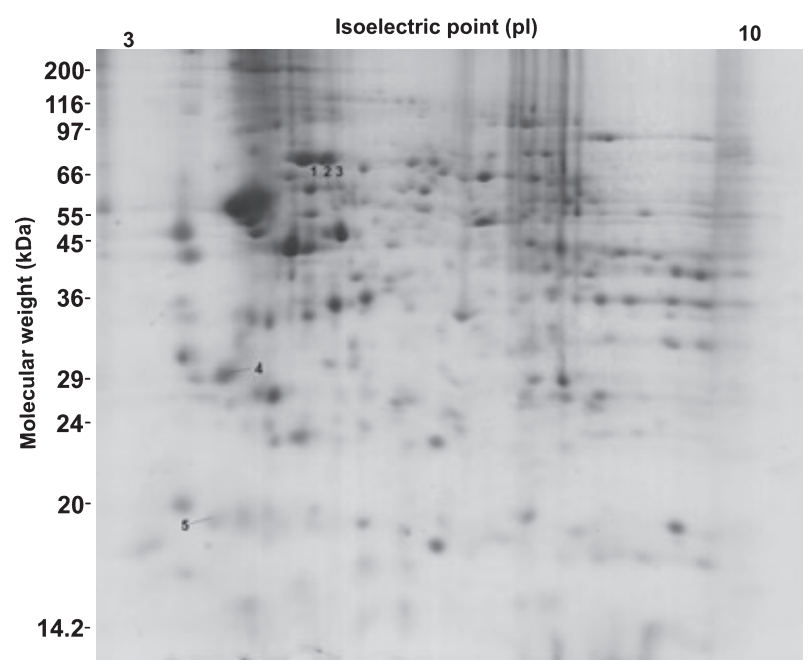

Fig. 3 Representative two-dimensional map of rat brain proteins. Proteins were extracted, solubilized, and separated on a pH 3-10 nonlinear immobilized $\mathrm{pH}$ gradient strip, followed by a 8-16\% polyacrylamide gel electrophoresis, as described in Materials and methods. After electrophoresis the gels were stained with Coomassie blue and the signed spots were analyzed by matrix-assisted laser desorption ionization mass spectroscopy. Spot numbers refer to Table 1.

sie blue-stained gel (Fig. 3). Blue-stained spots indicated by arrows and numbers in Fig. 3 and in Table 1 were selected for mass spectral identification by the merging of image analysis. Some spots gave no confident identification by the peptide mass fingerprinting procedure. Additional data were provided by nanoLC/MS/MS experiments. The peptide mixtures were fractionated by nanoHPLC and sequenced by tandem MS leading to the unambiguous identification of the protein candidate. Thirty different proteins were identified by MALDI-time of flight MS on the basis of mass fingerprint. In the NAcc of IR rats, expression level of two proteins, $14-3-3 \zeta / \delta$ protein and $\alpha$-syn, was significantly higher in comparison with the matched control $(p<0.05$, Student's $t$-test), while the expression level of heat shock protein 60 (HSP60) showed a significant decrease $(p<0.05$, Student's $t$-test) (Fig. 4). In the mPFC, 14-3-3 protein showed a slight, marginally significant $(p=0.0478$, Student's $t$-test) decrease with respect to group-reared controls (Fig. 4), while other protein expression levels were unaffected.

\section{Correlations between behavioral and neurochemical results in IR rats}

We first analyzed the correlation between startle amplitude values and the accumbal levels of $\alpha$-syn, 14-3-3 $\zeta / \delta$ protein, and HSP60 proteins in IR rats. Linear regression analysis revealed no significant correlations between startle values and levels of any of these proteins (data not shown). The regression analysis of PPI and $\alpha$-syn levels in the NAcc in IR 


\begin{tabular}{lllllll}
\hline $\begin{array}{l}\text { 2DE spot } \\
\text { number }\end{array}$ & $\begin{array}{l}\text { Protein } \\
\text { name }\end{array}$ & $\begin{array}{l}\text { Accession } \\
\text { number }\end{array}$ & Mr (KDa) & pl & $\begin{array}{l}\text { Sequence } \\
\text { Coverage (\%) }\end{array}$ & $\begin{array}{l}\text { Mascot } \\
\text { Score }\end{array}$ \\
\hline 1 & HSP60 & P63039 & 61 & 5.91 & 26 & 165 \\
2 & HSP60 & P63039 & 61 & 5.91 & 25 & 144 \\
3 & HSP60 & P63039 & 61 & 5.91 & 23 & 130 \\
4 & $14-3-3 \zeta / \delta$ & P63102 & 27.7 & 4.73 & 54 & 150 \\
5 & $\alpha-S y n u c l e i n$ & P37377 & 14.5 & 4.74 & 51 & 82
\end{tabular}

Table 1 Rat brain proteins with altered levels after IR treatment, identified by mass spectrometry

HSP60, heat shock protein 60; pl, isoelectric point; Mr, molecular weight; IR, isolation reared; 2DE, two-dimensional electrophoresis.

(a)

14-3-3 $\zeta$ - mPFC

SR

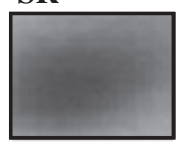

IR
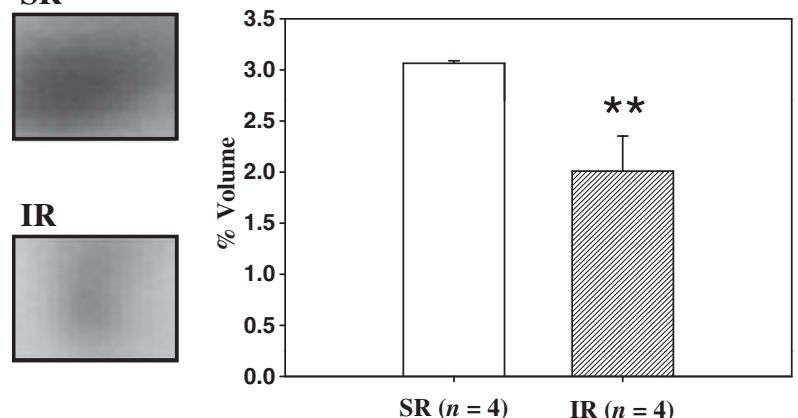

$\alpha$-synuclein - mPFC

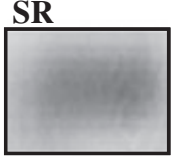

IR
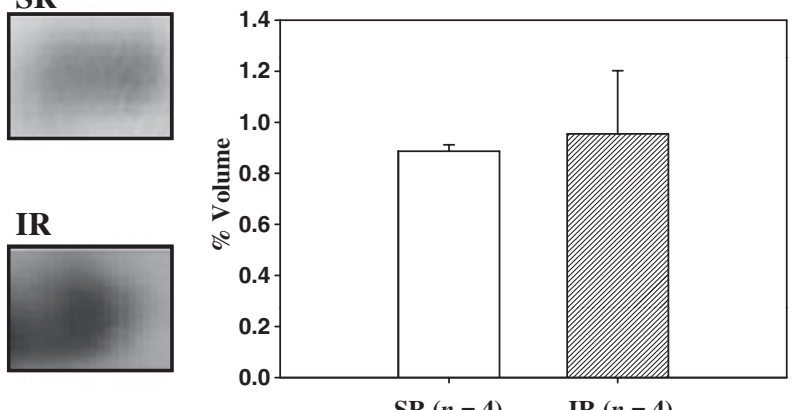

HSP60 - mPFC

SR

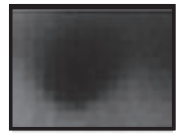

IR

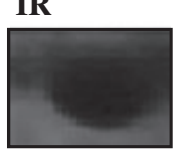

\section{.}

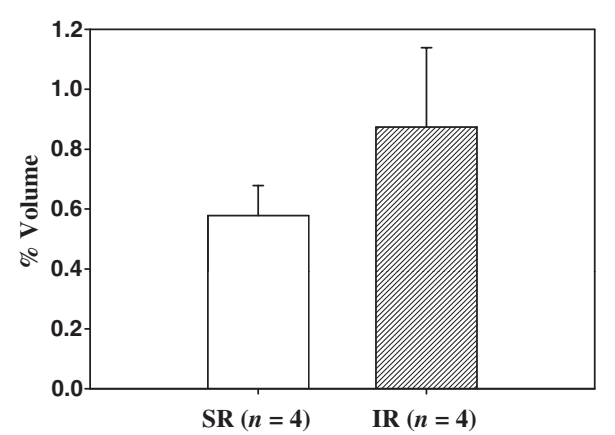

(b)

14-3-3 $\zeta$ - Nucleus accumbens

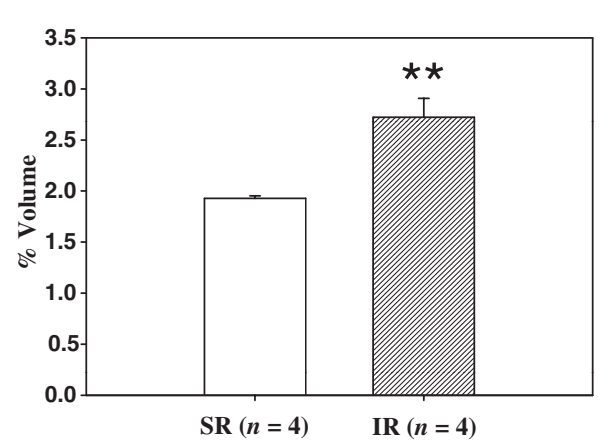

SR

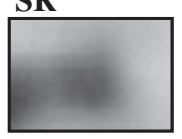

IR

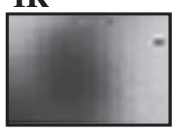

$\alpha$-synuclein - Nucleus accumbens

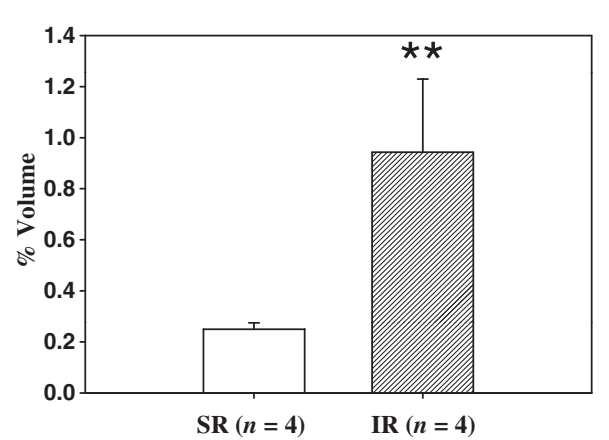

SR

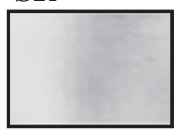

IR

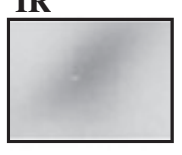

HSP60 - Nucleus accumbens

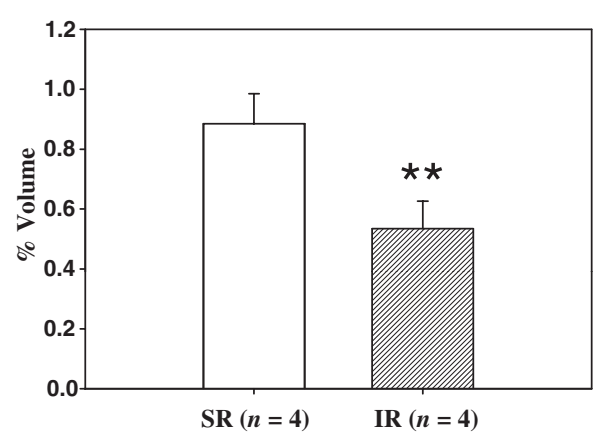

SR

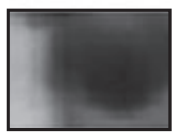

IR

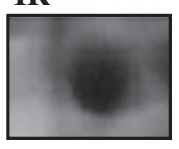

Fig. 4 Variation in expression level of three different proteins, 14-3-3 $\zeta / \delta$ protein, $\alpha$-synuclein ( $\alpha$-syn), and heat shock protein 60 (HSP60) in the medial prefrontal cortex (mPFC) and nucleus accumbens of isolation-reared (IR) and socially reared (SR) rats. ${ }^{* *} p<0.05$ (Student's $t$-test). 
(a)

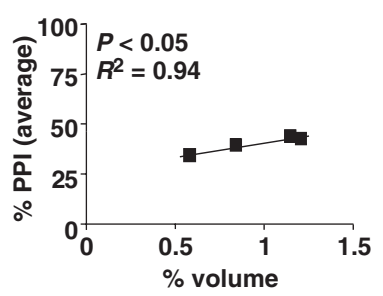

(c)

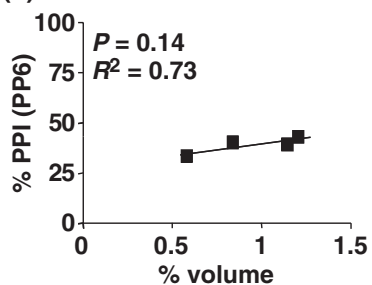

(b)

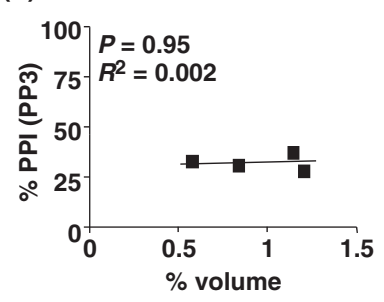

(d)

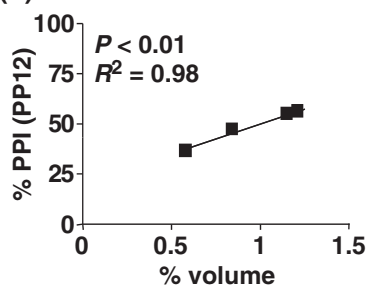

Fig. 5 Regression plot of the correlation between accumbal levels of $\alpha$-synuclein and (a) average \%PPI, (b) PP3-induced PPI, (c) PP6induced PPI and (d) PP12-induced PPI in isolation-reared rats. PPI, pre-pulse inhibition.

(a)

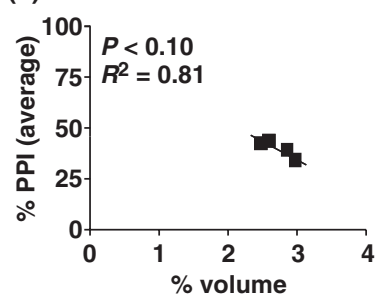

(c)

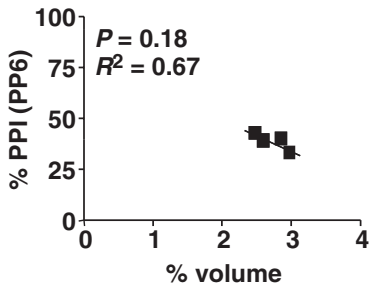

(b)

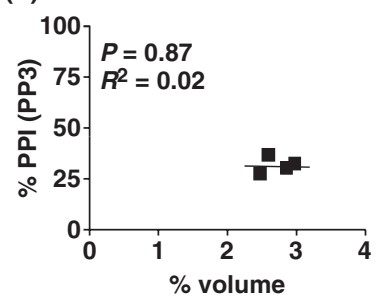

(d)

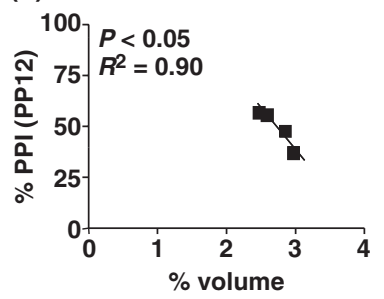

Fig. 6 Regression plot of the correlation between accumbal levels of 14-3-3 $\zeta / \delta$ protein and (a) average \%PPI, (b) PP3-induced PPI, (c) PP6-induced PPI and (d) PP12-induced PPI in isolation-reared rats. $\mathrm{PPI}$, pre-pulse inhibition.

rats revealed that the levels of this protein were significantly correlated with the overall variation in average PPI values $\left[F_{(1,2)}=29.87, p<0.05, \mathrm{R}^{2}=0.94\right]$ and PPI elicited by $82 \mathrm{~dB}$ (PP12) $\left[F_{(1,2)}=122.6, p<0.01, \mathrm{R}^{2}=0.98\right]$, but not with the other PPIs induced by either 73 (PP3) or $76 \mathrm{~dB}$ (PP6) (Fig. 5). We also identified a statistical trend for the correlation between 14-3-3 $\zeta / \delta$ and average PPI values $(p<0.10)$ in IR rats, which probably reflected a significant

correlation with PPI elicited by PP12 $\left[F_{(1,2)}=18.59\right.$, $\left.p<0.05, \mathrm{R}^{2}=0.90\right]$ but not by other pre-pulse levels (Fig. 6). No significant correlation was found between HSP60 protein levels and PPI in IR rats (data not shown). Finally, parallel analyses on the correlations between protein levels in mPFC and behavioral data did not yield any significant result (data not shown).

\section{Discussion}

In this study, we showed that under our experimental conditions IR elicited profound PPI deficits in IR SpragueDawley rats. These results are in keeping with numerous lines of evidence documenting gating impairments following this manipulation (Geyer et al. 1993; Cilia et al. 2001; but see Domeney and Feldon 1998 for contrasting results), together with other behavioral, morphological, and neurochemical abnormalities which strongly resembles core endophenotypes of schizophrenia (for a review, see Fone and Porkess 2008). Furthermore, IR rats exhibited a significant decrement in startle amplitude and no changes in startle habituation and latency. These findings are partially at variance with previous reports showing enhanced startle reflex (Krebs-Thomson et al. 2001). While the reasons of these apparent discrepancies remain unknown, they may reflect subtle differences in the conditions of isolation and startle testing as well as the rat strain. Similar variations may also account for the numerous inconsistencies in the effects of IR on both parameters (Varty and Geyer 1998; Varty et al. 1999).

Proteomic analyses revealed significant increases in $\alpha$-syn and 14-3-3 $\zeta / \delta$ proteins as well as a decrease in HSP60 in the NAcc of a representative subgroup of IR rats when compared with SR controls. Notably, changes in $\alpha$-syn and 14-3-3 $\zeta / \delta$ proteins in IR rats are significantly correlated with variations in intensity-specific PPIs, probably signifying a role of these proteins in the pathophysiology of IR-induced gating deficits.

Regression analysis revealed a direct correlation between accumbal levels of 14-3-3 $\zeta / \delta$ proteins and PP12-elicited PPI in IR rats. This association was not paralleled by significant correlations between startle amplitudes and protein levels. The family of 14-3-3 proteins has been shown to serve a number of critical functions in the regulation of many critical signal transduction pathways, by interacting with protein kinases (Aitken et al. 1990; McPherson et al. 1999) and other enzymes (for a review, see Aitken 2002). In consideration of the role of 14-3-3 proteins as pleiotropic effectors of cell physiology and signaling, it is not possible to exactly predict the significance of the enhancement in 14-3-3 $\zeta / \delta$ protein levels observed in the NAcc IR rats and its role in gating regulation. Nevertheless, previous evidence on the involvement of 14-3-3 in synaptic vesicles transport and exocytosis (Broadie et al. 1997; Roth et al. 1999; Lotharius and Brundin 2002a) is in accord with previous evidence 
indicating pre-synaptic dysfunctions in the striatum of IR rats (Hall et al. 1998; Barr et al. 2004). Specifically, the increase in 14-3-3 proteins may reflect enhanced DA production in NAcc as these molecules have been shown to bind and activate tyrosine hydroxylase (Ichimura et al. 1988; Itagaki et al. 1999), the rate-limiting enzyme in DA synthesis. Even though no direct measure of DArgic system activity is present in our study, this possibility is consistent with previous studies documenting impairments in DArgic signaling in the NAcc of IR rats (Jones et al. 1990; Fulford and Marsden 1998) as well as the role of accumbal DA in gating impairments in IR rats (Powell et al. 2003).

The levels of $\alpha$-syn in NAcc of IR rats were significantly correlated (positively and negatively, respectively) with average values of PPI and PPI elicited by $82 \mathrm{~dB}$ pre-pulses (12 dB above the background noise). Although the functional role of $\alpha$-syn is still largely elusive, emerging findings indicate that this molecule shares physical and functional homology with 14-3-3 proteins (Ostrerova et al. 1999). Accordingly, $\alpha$-syn also participates in a number of intracellular signaling pathways and binds to a broad array of enzymes (Gallardo et al. 2008). The link between the fourfold increase in $\alpha$-syn and IR-caused PPI deficits may be based on the role of this protein in synaptic dysfunctions. For instance, $\alpha$-syn exerts a negative control of synaptic vesicle replenishment with several neurotransmitters (Abeliovich et al. 2000; Yavich et al. 2004), and its deficiency results in a markedly reduced number of synaptic vesicles in the resting pool (Cabin et al. 2002) and increased number of readily releasable vesicles (Senior et al. 2008). Concurrently, alterations in $\alpha$-syn have been shown to affect the function of several neurotransmitters, such as serotonin, norepinephrine, and glutamate (Wersinger et al. 2006a,b; Gureviciene et al. 2007) which play important roles in the behavioral outcomes of postweaning isolation in rats (Fulford and Marsden 1997, 1998; Lapiz et al. 2003; Melendez et al. 2004). Notably, $\alpha$-syn has also been shown to play a key role in the modulation of DArgic activity (Lotharius and Brundin 2002a,b; Sidhu et al. 2004), and its over-expression in vivo has been associated with higher DA synthesis rate (Caudle et al. 2007; Alerte et al. 2008). Furthermore, $\alpha$-syn has been shown to reduce DA re-uptake by a negative modulation of the membrane DA transporter (Wersinger and Sidhu 2003 Sidhu et al. 2004) and the vesicular monoamine transporter 2 , which result in increased amphetamine-induced DA release (Lotharius et al. 2002). It is worth noting that analogously enhanced neurochemical and behavioral responses to amphetamine have been observed in IR animals (Jones et al. 1990; Hall et al. 1998).

The increased expression of $\alpha$-syn and 14-3-3 proteins may also reflect the existence of a neurodegenerative process. In fact, these molecules have been involved in the pathogenesis of numerous neurodegenerative disorders (Spillantini and Goedert 2000; Dougherty and Morrison 2004), and preliminary evidence has actually established that chronic stress and social isolation can predispose to or induce neurodegenerative alterations in brain (Bartesaghi and Severi 2004; Conrad et al. 2004).

Although the levels of HSP60 did not exhibit any correlation with the behavioral data in IR rats, it is possible that its decreased expression might also signify a reduction in neuroprotective mechanisms, as this protein has been shown to act as a molecular chaperon that plays an essential role for recovery from stress-induced protein damage (Hartl and Hayer-Hartl 2002). In general, HSPs are critical elements in the cellular response to unfolded proteins and in promoting proper protein folding to prevent aggregation as well as in promoting ubiquitination and degradation of misfolded proteins (Fink 1999). Accordingly, recent evidence indicates that HSP proteins may also play a role in reducing $\alpha$-syn aggregation and toxicity (Klucken et al. 2004).

In conclusion, our findings suggest that the PPI deficits induced by IR may be caused by imbalances in 14-3-3 and $\alpha$-syn expression in the NAcc. PPI has been extensively validated as a dependable paradigm to measure integrity of gating functions and pre-attentional filtering (Braff et al. 2001; Swerdlow et al. 2001), and its translational salience for the experimental study of gating functions is supported by the similar testing modalities between humans and other vertebrates. Furthermore, spontaneous PPI deficits are observed in schizophrenia and other psychiatric disorders featuring gating dysfunctions, supporting the validity of this paradigm as a dependable tool for the study of psychosis (Braff et al. 2001). Accordingly, PPI disruption is triggered by psychotomimetic substances and restored by antipsychotic agents in both humans and animals (Mansbach and Geyer 1989; Geyer et al. 1990; Johansson et al. 1995; Geyer 1998; Martinez et al. 1999). Based on these premises, the identification of molecular markers correlated with IRinduced PPI deficits holds great relevance for the study of the pathophysiology of psychotic disorders and gating dysfunctions, and warrants further studies on the role of $\alpha$-syn, HSP60, and 14-3-3 proteins in schizophrenia.

\section{References}

Abeliovich A., Schmitz Y., Fariñas I. et al. (2000) Mice lacking alphasynuclein display functional deficits in the nigrostriatal dopamine system. Neuron 25, 239-252.

Aitken A. (2002) Functional specificity in 14-3-3 isoform interactions through dimer formation and phosphorylation. Chromosome location of mammalian isoforms and variants. Plant Mol. Biol. 50, 993-1010.

Aitken A., Ellis C. A., Harris A., Sellers L. A. and Toker A. (1990) Kinase and neurotransmitters. Nature 344, 594.

Alerte T. N., Akinfolarin A. A., Friedrich E. E., Mader S. A., Hong C. S. and Perez R. G. (2008) Alpha-synuclein aggregation alters tyrosine hydroxylase phosphorylation and immunoreactivity: lessons from viral transduction of knockout mice. Neurosci. Lett. 435, 24-29. 
Bakshi V. P., Swerdlow N. R., Braff D. L. and Geyer M. A. (1998) Reversal of isolation rearing-induced deficits in prepulse inhibition by seroquel and olanzapine. Biol. Psychiatry 43, 436-445.

Barr A. M., Young C. E., Sawada K., Trimble W. S., Phillips A. G. and Honer W. G. (2004) Abnormalities of presynaptic protein CDCrel1 in striatum of rats reared in social isolation: relevance to neural connectivity in schizophrenia. Eur. J. Neurosci. 20, 303-307.

Bartesaghi R. and Severi S. (2004) Effects of early environment on field CA2 pyramidal neurons in the guinea-pig. Neuroscience 123, 703714.

Binder E. B., Kinkead B., Owens M. J., Kilts C. D. and Nemeroff C. B. (2001) Enhanced neurotensin neurotransmission is involved in the clinically relevant behavioral effects of antipsychotic drugs: evidence from animal models of sensorimotor gating. J. Neurosci. 21, 601-608.

Braff D. L., Grillon C. and Geyer M. A. (1992) Gating and habituation of the startle reflex in schizophrenic patients. Arch. Gen. Psychiatry 49, 206-215.

Braff D. L., Geyer M. A. and Swerdlow N. R. (2001) Human studies of prepulse inhibition of startle: normal subjects, patient groups, and pharmacological studies. Psychopharmacology 156, 234-258.

Brenes J. C., Rodríguez O. and Fornaguera J. (2008) Differential effect of environment enrichment and social isolation on depressive-like behavior, spontaneous activity and serotonin and norepinephrine concentration in prefrontal cortex and ventral striatum. Pharmacol. Biochem. Behav. 89, 85-93.

Broadie K., Rushton E., Skoulakis E. M. and Davis R. L. (1997) Leonardo, a Drosophila 14-3-3 protein involved in learning, regulates presynaptic function. Neuron 19, 391-402.

Cabin D. E., Shimazu K., Murphy D. et al. (2002) Synaptic vesicle depletion correlates with attenuated synaptic responses to prolonged repetitive stimulation in mice lacking alpha-synuclein. J. Neurosci. 22, 8797-8807.

Carrette O., Burkhard P. R., Sanchez J. C. and Hochstrasser D. F. (2006a) State-of-the-art two-dimensional gel electrophoresis: a key tool of proteomics research. Nat. Protoc. 1, 812-823.

Carrette O., Burkhard P. R., Hochstrasser D. F. and Sanchez J. C. (2006b) Age-related proteome analysis of the mouse brain: a 2-DE study. Proteomics 6, 4940-4949.

Caudle W. M., Richardson J. R., Wang M. Z., Taylor T. N., Guillot T. S., McCormack A. L., Colebrooke R. E., Di Monte D. A., Emson P. C. and Miller G. W. (2007) Reduced vesicular storage of dopamine causes progressive nigrostriatal neurodegeneration. J. Neurosci. 27, $8138-8148$.

Cilia J., Reavill C., Hagan J. J. and Jones D. N. (2001) Long-term evaluation of isolation-rearing induced prepulse inhibition deficits in rats. Psychopharmacology 156, 327-337.

Conrad C. D., Jackson J. L. and Wise L. S. (2004) Chronic stress enhances ibotenic acid-induced damage selectively within the hippocampal CA3 region of male, but not female rats. Neuroscience 125, 759-767.

Domeney A. and Feldon J. (1998) The disruption of prepulse inhibition by social isolation in the Wistar rat: how robust is the effect? Pharmacol. Biochem. Behav. 59, 883-890.

Dougherty M. K. and Morrison D. K. (2004) Unlocking the code of 14-3-3. J. Cell Sci. 117, 1875-1884.

Einon D. F. and Morgan M. J. (1977) A critical period for social isolation in the rat. Dev. Psychobiol. 10, 123-132.

Fink A. L. (1999) Chaperone-mediated protein folding. Physiol. Rev. 79 , 425-449.

Fone K. C. F. and Porkess M. V. (2008) Behavioural and neurochemical effects of post-weaning social isolation in rodents relevance to developmental neuropsychiatric disorders. Neurosci. Biobehav. 32, 1087-1102.
Fountoulakis M. (2004) Application of proteomics technologies in the investigation of the brain. Mass Spectrom. Rev. 23, 231-258.

Fountoulakis M., Schuller E., Hardmeier R., Berndt P. and Lubec G. (1999) Rat brain proteins: two-dimensional protein database and variations in the expression level. Electrophoresis 20, 3572-3579.

Fountoulakis M., Tsangaris G. T., Maris A. and Lubec G. (2005) The rat brain hippocampus proteome. J. Chromatogr. B Analyt. Technol. Biomed. Life Sci. 819, 115-129.

Fulford A. J. and Marsden C. A. (1997) Effect of isolation-rearing on noradrenaline release in rat hypothalamus and hippocampus in vitro. Brain Res. 748, 93-99.

Fulford A. J. and Marsden C. A. (1998) Effect of isolation-rearing on conditioned dopamine release in vivo in the nucleus accumbens of the rat. J. Neurochem. 70, 384-390.

Gallardo G., Schlüter O. M. and Südhof T. C. (2008) A molecular pathway of neurodegeneration linking alpha-synuclein to ApoE and Abeta peptides. Nat. Neurosci. 11, 301-308.

Garzón J. and Del Río J. (1981) Hyperactivity induced in rats by longterm isolation: further studies on a new animal model for the detection of antidepressants. Eur. J. Pharmacol. 74, 287-294.

Geyer M. A. (1998) Behavioral studies of hallucinogenic drugs in animals: implications for schizophrenia research. Pharmacopsychiatry Suppl. 2, 73-79.

Geyer M. A., Swerdlow N. R., Mansbach R. S. and Braff D. L. (1990) Startle response models of sensorimotor gating and habituation deficits in schizophrenia. Brain Res. Bull. 25, 485-498.

Geyer M. A., Wilkinson L. S., Humby T. and Robbins T. W. (1993) Isolation rearing of rats produces a deficit in prepulse inhibition of acoustic startle similar to that in schizophrenia. Biol. Psychiatry 34, 361-372.

Gorg A., Weiss W. and Dunn M. J. (2004) Current two-dimensional electrophoresis technology for proteomics. Proteomics 4, 36653685 .

Gureviciene I., Gurevicius K. and Tanila H. (2007) Role of alpha-synuclein in synaptic glutamate release. Neurobiol. Dis. 28, 83-89.

Hall F. S., Wilkinson L. S., Humby T., Inglis W., Kendall D. A., Marsden C. A. and Robbins T. W. (1998) Isolation rearing in rats: pre- and postsynaptic changes in striatal dopaminergic systems. Pharmacol. Biochem. Behav. 59, 859-872.

Hartl F. U. and Hayer-Hartl M. (2002) Molecular chaperones in the cytosol: from nascent chain to folded protein. Science 295, 1852 1858.

Heffner T. G., Hartman J. A. and Seiden L. S. (1980) A rapid method for the regional dissection of the rat brain. Pharmacol. Biochem. Behav. 13, 453-456.

Ichimura T., Toshiaki I., Okuyama T., Takahashi N., Araki K., Kuwano R. and Takahashi Y. (1988) Molecular cloning of cDNA coding for brain-specific 14-3-3 protein, a protein kinase-dependent activator of tyrosine and tryptophan hydroxylase. Proc. Natl Acad. Sci. USA 85, 7084-7088.

Itagaki C., Isobe T., Taoka M. et al. (1999) Stimulus-coupled interaction of tyrosine hydroxylase with 14-3-3 proteins. Biochemistry 38, $15673-15680$.

Johansson C., Jackson D. M., Zhang J. and Svensson L. (1995) Prepulse inhibition of acoustic startle, a measure of sensorimotor gating: effects of antipsychotics and other agents in rats. Pharmacol. Biochem. Behav. 52, 649-654.

Johnston-Wilson N. L., Sims C. D., Hofmann J. P., Anderson L., Shore A. D., Torrey E. F. and Yolken R. H. (2000) Disease-specific alterations in frontal cortex brain proteins in schizophrenia, bipolar disorder, and major depressive disorder. The Stanley Neuropathology Consortium. Mol. Psychiatry 5, 142-149.

Jones G. H., Marsden C. A. and Robbins T. W. (1990) Increased sensitivity to amphetamine and reward-related stimuli following social 
isolation in rats: possible disruption of dopamine-dependent mechanisms of the nucleus accumbens. Psychopharmacology 102, 364-372.

Klucken J., Shin Y., Masliah E., Hyman B. T. and McLean P. J. (2004) Hsp70 reduces $\alpha$-synuclein aggregation and toxicity. J. Biol. Chem. 279, 25497-25502.

Krebs-Thomson K., Giracello D., Solis A. and Geyer M. A. (2001) Postweaning handling attenuates isolation-rearing induced disruptions of prepulse inhibition in rats. Behav. Brain Res. 120, 221-224.

Lapiz M. D., Fulford A., Muchimapura S., Mason R., Parker T. and Marsden C. A. (2003) Influence of postweaning social isolation in the rat on brain development, conditioned behavior, and neurotransmission. Neurosci. Behav. Physiol. 33, 13-29.

Lotharius J. and Brundin P. (2002a) Impaired dopamine storage resulting from alpha-synuclein mutations may contribute to the pathogenesis of Parkinson's disease. Hum. Mol. Genet. 11, 2395-2407.

Lotharius J. and Brundin P. (2002b) Pathogenesis of Parkinson's disease: dopamine, vesicles and $\alpha$-synuclein. Nat. Rev. Neurosci. 3, 932942.

Lotharius J., Barg S., Wiekop P., Lundberg C., Raymon H. K. and Brundin P. (2002) Effect of mutant alpha-synuclein on dopamine homeostasis in a new human mesencephalic cell line. J. Biol. Chem. 277, 38884-38894.

Mansbach R. S. and Geyer M. A. (1989) Effects of phencyclidine and phencyclidine biologs on sensorimotor gating in the rat. Neuropsychopharmacology 2, 299-308.

Martinez Z. A., Ellison G. D., Geyer M. A. and Swerdlow N. R. (1999) Effects of sustained cocaine exposure on sensorimotor gating of startle in rats. Psychopharmacology 142, 253-260.

McPherson R. A., Harding A., Roy S., Lane A. and Hancock J. F. (1999) Interactions of c-Raf-1 with phosphatidylserine and 14-3-3. Oncogene 18, 3862-3869.

Melendez R. I., Gregory M. L., Bardo M. T. and Kalivas P. W. (2004) Impoverished rearing environment alters metabotropic glutamate receptor expression and function in the prefrontal cortex. Neuropsychopharmacology 29, 1980-1987.

Ostrerova N., Petrucelli L., Farrer M., Mehta N., Choi P., Hardy J. and Wolozin B. (1999) Alpha-synuclein shares physical and functional homology with 14-3-3 proteins. J. Neurosci. 19, 5782-5791.

Peters Y. M. and O'Donnell P. (2005) Social isolation rearing affects prefrontal cortical response to ventral tegmental area stimulation. Biol. Psychiatry 57, 1205-1208.

Powell S. B., Geyer M. A., Preece M. A., Pitcher L. K., Reynolds G. P. and Swerdlow N. R. (2003) Dopamine depletion of the nucleus accumbens reverses isolation-induced deficits in prepulse inhibition in rats. Neuroscience 119, 233-240.

Roth D., Birkenfeld J. and Betz H. (1999) Dominant-negative alleles of 14-3-3 proteins cause defects in actin organization and vesicle targeting in the yeast Saccharomyces cerevisiae. FEBS Lett. 460, $411-416$

Senior S. L., Ninkina N., Deacon R., Bannerman D., Buchman V. L., Cragg S. J. and Wade-Martins R. (2008) Increased striatal dopamine release and hyperdopaminergic-like behaviour in mice lacking both alpha-synuclein and gamma-synuclein. Eur. J. Neurosci. 27, 947-957.

Sidhu A., Wersinger C. and Vernier P. (2004) Does $\alpha$-synuclein modulate dopaminergic synaptic content and tone at the synapse? FASEB $J$. 18, 637-647.

Spillantini M. G. and Goedert M. (2000) The alpha-synucleinopathies: Parkinson's disease, dementia with Lewy bodies, and multiple system atrophy. Ann. NY Acad. Sci. 920, 16-27.

Swerdlow N. R., Braff D. L., Taaid N. and Geyer M. A. (2001) Assessing the validity of an animal model of deficient sensorimotor gating in schizophrenic patients. Arch. Gen. Psychiatry 51, 139-154.

Teunissen C. E. and Scheltens P. (2007) Use of proteomic approaches to identify disease biomarkers. Lancet Neurol. 6, 1036-1037.

Varty G. B. and Geyer M. A. (1998) Effects of isolation rearing on startle reactivity, habituation, and prepulse inhibition in male Lewis, Sprague-Dawley, and Fischer F344 rats. Behav. Neurosci. 112, $1450-1457$.

Varty G. B., Braff D. L. and Geyer M. A. (1999) Is there a critical developmental 'window' for isolation rearing-induced changes in prepulse inhibition of the acoustic startle response? Behav. Brain Res. 100, 177-183.

Weiss I. C. and Feldon J. (2001) Environmental animal models for sensorimotor gating deficiencies in schizophrenia: a review. Psychopharmacology 156, 305-326.

Wersinger C. and Sidhu A. (2003) Attenuation of dopamine transporter activuty by $\alpha$-synuclein. Neurosci. Lett. 340, 189-192.

Wersinger C., Jeannotte A. and Sidhu A. (2006a) Attenuation of the norepinephrine transporter activity and trafficking via interactions with alpha-synuclein. Eur. J. Neurosci. 24, 3141-3152.

Wersinger C., Rusnak M. and Sidhu A. (2006b) Modulation of the trafficking of the human serotonin transporter by human alphasynuclein. Eur. J. Neurosci. 24, 55-64.

Yavich L., Tanila H., Vepsalainen S. and Jakala P. (2004) Role of $\alpha$-synuclein in presynaptic dopamine recruitment. J. Neurosci. 24, 11165-11170. 